IOSR Journal of Pharmacy

Mar.-Apr. 2012, Vol. 2(2) pp: 225-227

\title{
SPONTANEOUS ORBITAL EMPHYSEMA
}

\author{
Dr Vikas Sikarwar ${ }^{1}$, Dr R.S Bisht ${ }^{1}$, Dr Anshuman Darbari. ${ }^{2}$ \\ ${ }^{I}$ Dept. E.N.T and Head \& Neck Surgery; Veer Chandra Singh Garhwali Govt Medical Science and Research Institute \\ Srinagar, UK, India \\ ${ }^{2}$ Department of Surgery; Veer Chandra Singh Garhwali Govt Medical Science and Research Institute Srinagar, UK, India
}

\begin{abstract}
Orbital emphysema due to barotrauma is rare ocular emergency which is usually benign but can cause serious visual complications if not timely recognized. We report a case of orbital emphysema in 34-years-old male presented with sudden onset of right periorbital swelling after forceful blowing of the nose. There was no history of facial trauma or previous surgery. Examination of the patient revealed crepitant eyelid swelling and ptosis but no visual impairment or diplopia was seen. Otorhinolaryngologic examination revealed clotted blood in right nasal cavity. Computerized tomography demonstrated areas of emphysema in the right orbit. Patient was managed conservatively with favorable outcome. The purpose of this presentation is to stress the recognition of rare possibility of orbital emphysema in the absence of trauma as timely intervention can prevent vision loss.
\end{abstract}

\section{Keywords: Barotrauma, Orbital Emphysema, Nose blowing.}

\section{INTRODUCTION}

Trauma is the most frequent cause of orbital emphysema however, orbital emphysema may also occur spontaneously as a complication of barotrauma, infection and surgery ${ }^{1}$. In most cases, orbital emphysema resolves spontaneously without compromising the ocular function if excessive amount of air accumulate within the orbit, however, complications such as occlusion of the central retinal artery or compressive optic neuropathy may lead to loss of vision if not recognized and treated promptly.

\section{CASE REPORT}

A 34 year male was presented in ENT OPD with periorbital swelling of sudden onset (figure 1). History of Patient revealed an episode of unprovoked epistaxis and thereafter patient tried to clear off blood clots from his nose for which he forcefully blew his nose which resulted in the sudden periorbital swelling over right side. There was no history of sinusitis, facial trauma or previous surgery. On examination, vision, eye movements and pupillary light reflexes were found normal. Computerized tomography (CT) revealed massive subcutaneous emphysema with extraconal air in the right superior orbit without extension to the optic foramen. Scan also revealed proptosis and a blowout fracture of the medial wall of the right orbit (Figure 2). Patient was managed conservatively with decongestant nasal drops, analgesics and prophylactic antibiotics. Patient was kept in observation for 24 hours and then was discharged on medications and with strict instructions not to blow his nose. Patient returned after a week with complete resolution of orbital emphysema (Figure 3).

\section{DISCUSSION}

Orbital emphysema is a complication of orbital fracture which usually results from direct injury. ${ }^{1}$ A few cases of orbital emphysema have been reported due to indirect injury like nose blowing ${ }^{2}$, post surgical sneezing and pressure change during air travel ${ }^{3}$. Orbital emphysema mostly arises from fracture of lamina papyracea of the ethmoids and maxillary roof. Emphysema in the eye region is found in three sites - (a) Eye lids (b) orbit (c) lids \& orbit combined. Only lid emphysema is rare and is due to fracture of the lacrimal bone anterior to the tarso-orbital fascia causing rupture of lacrimal sac allowing air to escape from nasal cavity in to tissue of lids. True orbital emphysema is due to fracture of the bony walls of the orbit involving the paransal sinuses usually ethmoid or maxillary antrum and, less commonly the frontal sinuses. Air does not enter spontaneously following fracture but intermittently as pressure in the respiratory passage is raised acutely ${ }^{4}$, which happens after nose blowing, coughing, sneezing or emesis. A forceful expiratory effort can create significantly elevated intranasal pressure and fracture site acts as two-way valve so air can escape through this fracture site freely. However, serious orbital emphysema occurs with a small fracture site and Orbital tissue such as fat, falls back on this small communication site, blocking the exit of air and creating a one-way valve. Trapped intraorbital air mass increases resulting in compression of the vital structures within orbit and may result in vision loss. 


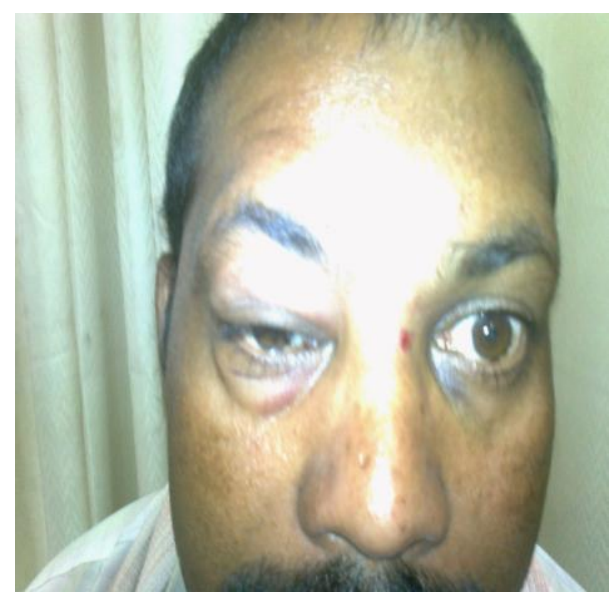

Fig 1 Right sided periorbital emphysema

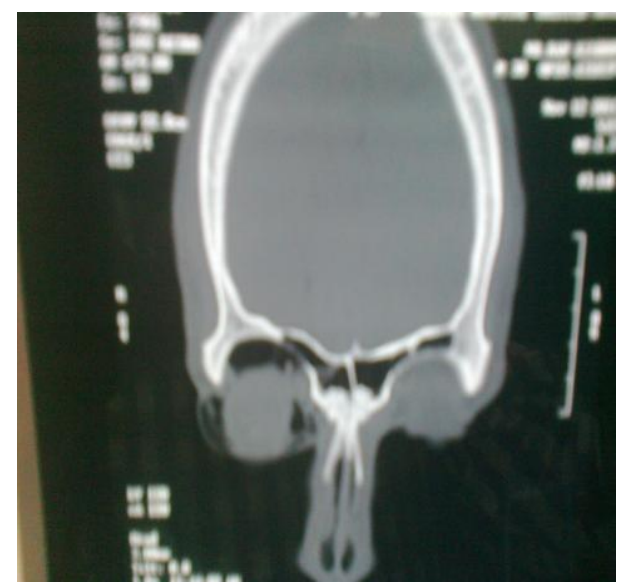

Fig.2.CT.scan.showing orbital Emphysema

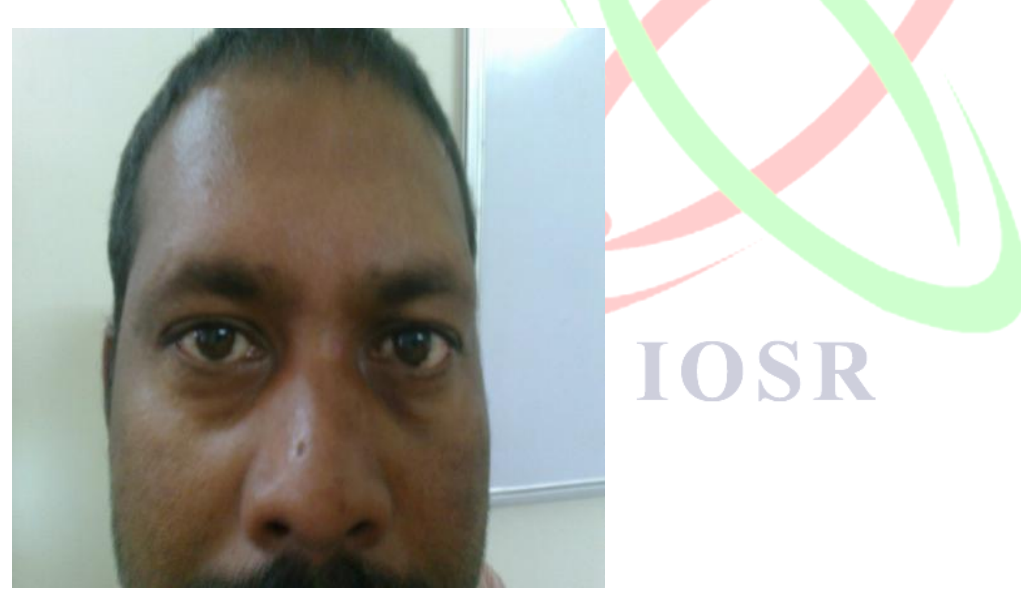

Fig 3 Complete resolution of orbital emphysema

The diagnosis of orbital emphysema is usually made by history alone, supported by results of clinical examination and confirmed with orbital CT scan. Most of cases resolve spontaneously in two to three weeks without any sequelae.

Management of most orbital emphysema includes close observation and conservative treatment with Nasal decongestants, antibiotics and steroids ${ }^{5}$. Instruction against nose blowing and valsalva is given. With this management alone, most of the cases resolve spontaneously in two to three weeks. ${ }^{2}$ If signs of ischemic optic neuritis, central artery occlusion and increased intraorbital pressure develops then immediate decompression can be achieved by syringe under saline ${ }^{5}$. In emergency cases, Quick decompression with lateral canthotomy /cantholysis is indicated. ${ }^{5}$ 


\section{CONCLUSION}

Orbital emphysema is an uncommon complication of orbital fracture following direct trauma or barotrauma. Although majority of these cases are asymptomatic and resolve without any adverse sequele, a close observation is must as timely intervention can prevent vision loss.

\section{REFERENCES}

1. Burm JS, Chung CH, Oh SJ. Pure orbital blowout fracture: New concepts and importance of medial orbital blowout fracture. Plast Reconstr Surg 1999;103:1839-1849.

2. Mohan B, Singh KP. Bilateral sub-cutaneous orbital emphysema oforbit following nose blowing. J Laryngol Otol 2001; 115: 319-20.

3. Monaghan AM, Millar BG. Orbital emphysema during air travel: a case report. J Craniomaxillofac Surg 2002;30(6):367-8.

4. $\quad$ Lloyd G :orbital emphysema.Br j radiol 39:933- 938- 1966.

5. Hunts JH, Patrinely JR, Holds JB, Anderson RL. Orbital emphysema. Staging and acute management. Ophthalmology 1994;101:960-966.

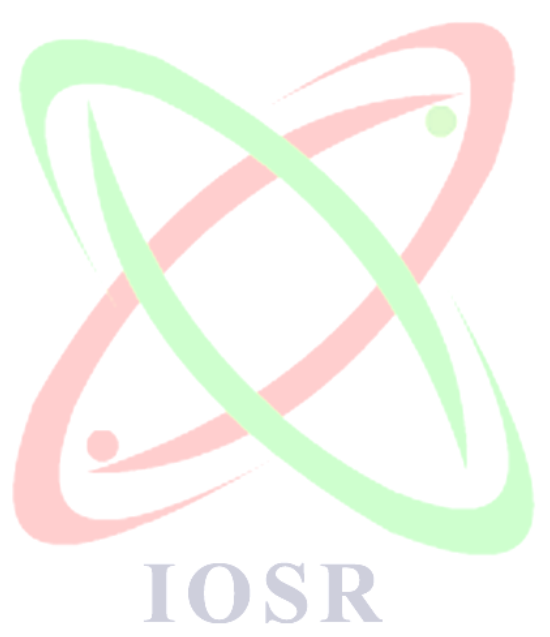

